\title{
Luminance effects on visual evoked brain responses to flash onset and offset
}

\author{
DAVID F. DINGES and DONALD I. TEPAS \\ Saint Louis University, Saint Louis, Missouri 63103
}

\begin{abstract}
Visual evoked brain responses (VEBRs) to the onset (ON VEBR) and offset (OFF VEBR) of square wave white light flashes presented monocularly were recorded from the scalp of three adult humans. Stimuli were present in Maxwellian view, and subtended a visual angle of $1.5 \mathrm{deg}$. Target luminance was varied in $.5 \mathrm{log}$ unit steps over a $4.0 \mathrm{log}$ unit range. For all subjects, the OFF VEBR was similar to the ON VEBR in appearance and latency, but smaller in amplitude. Both linear-log and log-log regression lines fit to the data indicate that peak latency of both ON and OFF VEBR measures decreases as a function of increasing luminance. Also, the amplitude of the VEBR increases as luminance increases. Comparable statistically significant $O N$ and $O F F$ VEBR regression lines are not significantly different in slope. These results do not support the notion that different neural systems mediate both ON and OFF VEBRs, thereby suggesting that the same or similar neural systems may mediate both VEBRs.
\end{abstract}

The existence of a cortical response to the offset or removal of a visual stimulus (OFF response) has been known for many years (Fischer, 1932). Despite this fact, only a few reports of the human averaged visual evoked brain response (VEBR) have considered the OFF response (Bartlett \& White, 1968; Clynes, Kohn, \& Lifshitz, 1964; Efron, 1964; Emrich \& Michael, 1970; Harter, 1971; Peacock, 1970; White \& Eason, 1966). In general, these reports suggest that the VEBR OFF response is similar but smaller than the response to visual stimulus onset ( $\mathrm{ON}$ response). Reliable detection of the OFF response is not always evident, and quantitative comparisons of VEBR ON and OFF measures are not common in these studies.

Luminance may provide a useful dimension for the more precise and direct comparison of $\mathrm{ON}$ and $\mathrm{OFF}$ VEBR characteristics. Only one study (Clynes et al., 1964) has compared ON and OFF VEBR measures while varying stimulus luminance. They report a curvilinear relationship between the amplitude of the ON VEBR and luminance with a linear relationship between the amplitude of the OFF VEBR and luminance. Tepas, Guiteras, \& Klingaman (1974) report linear relationships between luminance and both the amplitude and latency measures of ON VEBR waveforms. They have noted that precise visual stimulus control and response measurement are needed to obtain linear functions from $\mathrm{ON}$ VEBR measurements. Unfortunately, Clynes et al. (1964) present few details concerning their stimulation and measurement techniques. Consequently, there remains the clear possibility that the difference in $\mathrm{ON}$ and

The results reported are based on a thesis submitted by the first author to Saint Louis University in partial fulfillment of the MS degree. Requests for reprints should be sent to David F. Dinges, Child Neurology EEG Lab, The George Washington University Hospital, 901 23rd Street, N.W., Washington, D.C. 20037.
OFF intensity functions reported by them may not be representative. The present study reports $\mathrm{ON}$ and OFF VEBR measures obtained at nine luminance levels for each of three subjects under the same controlled experimental conditions. This allows direct comparison of $\mathrm{ON}$ and OFF VEBR intensity functions.

\section{METHOD}

\section{Subjects}

Three healthy normal adult humans, age 24 years, served as subjects. Except for corrected myopia, all subjects had normal color vision as tested by the Bausch and Lomb Ortho-Rater. Subjects were selected on the basis of their ability to produce recordable EEG under the experimental conditions.

\section{Apparatus}

Stimulation system. The stimulus system used was the same as that described by Tepas (1974). Visual stimuli were presented to the right eye of all subjects by way of a two-channed Maxwellian view optical system. Channel 1 of the system delivered a continuous blue surround target, while Channel 2 delivered a white flashing target. A tungsten ribbon-filament lamp served as the source for both channels. Channel 2 subtended a visual angle of $1.66 \mathrm{deg}$, while the constant blue surround circumscribing the white flash subtended a visual angle of $16 \mathrm{deg}$. The white target subjectively matched the blue surround when 3.0 log units of neutral density filtering were placed in the white target channel. Subjects were seated in a dental chair and maintained fixation by placing their teeth on a biteboard containing their dental impression.

The stimuli consisted of square wave white light flashes which were on for $1,034 \mathrm{msec}$ and off for $1,000 \mathrm{msec}$. The risefall time of the flash was $9 \mathrm{msec}$. Flash luminance was varied in nine $.5 \log$ unit steps over a four log unit range using fixed-value neutral density filters (Oriel). The maximum available retinal illuminance of target flash at $.0 \log$ was 180,000 trolands, as measured by a MacBeth illuminometer (Westheimer, 1966).

Recording system. The electroencephalogram (EEG) was recorded on one channel of a Grass Model 7 polygraph. The one-half amplitude high-frequency band pass set at $500 \mathrm{~Hz}$, while the low-frequency band pass set at $.15 \mathrm{~Hz}$. A bipolar EEG recording was made using Grass E5S silver-silver chloride elec- 
SUBUECT: CMD
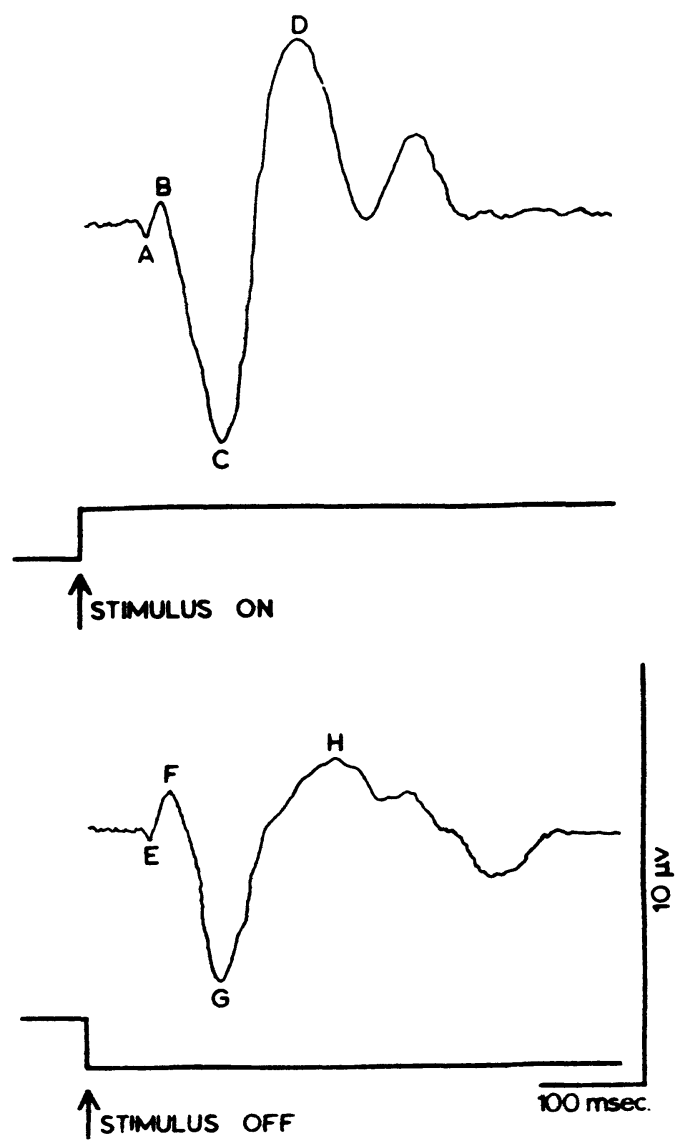

Figure 1. Typical VEBRs to onset and offset of a white flash stimulus $(.5 \mathrm{log})$. Peak deflections of ON and OFF VEBRs were arbitrarily labeled A, B, C, D, E, F, G, and H. Downward deflection indicates that the $\mathrm{O}_{z}$ electrode was positive with respect to the $C_{z}$ electrode.

trodes placed on $\mathrm{O}_{z}$ and $\mathrm{C}_{z}$ (Jasper, 1958). A Beckman 16-mm biopotential electrode attached to the forehead served as ground. The recording and computing systems were identical to those used by Tepas (1974), except for the addition of two Clare $\mathrm{Hg} 1004$ mercury wetted relays connected between two GrasonStadler 1222 output control modules and the AX08 peripheral of the Lab 8/I computer system (Schweitzer \& Tepas, 1974). A Digital Equipment Corporation Lab-8 computer system, using the Advanced Averager Program software tape (DEC-LB-U18C-PB), timebase averaged the EEG to yield the VEBR. Averaged VEBR waveforms consisted of 500 time points distributed over a 500-msec analysis time starting from either stimulus onset or offset. VEBR waveforms were displayed on an oscilloscope during averaging.

\section{Procedure}

Both ON and OFF VEBRs were collected for each of the nine intensities and one no-flash condition (control condition) in each session. One hundred EEG traces to onset and offset were averaged at each intensity during each of 10 sessions. Within a session, stimulus luminance was varied in a pseudorandom order, with no luminance value used more than once as the first trial of a session. Subjects were dark adapted in a shielded chamber $10 \mathrm{~min}$ before each session, and were given a 3-min rest period between each trial. After each trial, ON and OFF waveforms were recorded on punch paper tape for later analysis. Waveforms for each intensity within each subject were summed across sessions after all 10 sessions were completed using a software program developed in the lab (Tepas, Kress, \&
Klingaman, 1975). This resulted in one waveform for the 1,000 stimulus presentations for each stimulus luminance value. The summed waveforms were printed out on an ASR33 Teletype in decimal values. Both microvolt $(\mu \mathrm{v})$ and millisecond values were computed from these decimal values. A standard calibration square wave $(10 \mu \mathrm{v}$ and $100 \mathrm{msec})$ was also averaged each session, and provided a scale calibration of the waveform decimal values.

\section{RESULTS}

Figure 1 illustrates typical VEBR ON and OFF waveforms for Subject C.M.D. Peak measures were arbitrarily labeled in accordance with a nomenclature system previously used in this lab (Tepas, 1974), and have no implicit significance. These measures are identified in Figure 1 by the letters A, B, C, and D for the $\mathrm{ON}$ waveform and $\mathrm{E}, \mathrm{F}, \mathrm{G}$, and $\mathrm{H}$ for the OFF waveform. Amplitude was measured as the peak-to-peak distance between two deflections. Latency was measured as the time from stimulus onset or offset.

The ON waveforms conformed to the expected shape of waveforms previously reported by $\mathrm{C}_{\mathrm{z}}-\mathrm{O}_{\mathrm{z}}$ bipolar records under similar conditions (Tepas et al., 1974). The similarity in wave shape between ON and OFF responses from Subject C.M.D. for all luminance values used in the present study are shown in Figure 2. Each VEBR waveform is a product of 1,000 averaged EEG traces. Control trials (a constant blue surround but no flashing target) produced no detectable VEBR waveforms. As Figure 2 indicates, the OFF response was consistently smaller in amplitude than the ON response for Subject C.M.D. This was also true for Subjects P.A.K. and K.F.

Within each subject latency measures indicate that the OFF response deflections have latency ranges similar to the $\mathrm{ON}$ response deflections. Within each subject $\mathrm{ON}$ and OFF component latency ranges overlap, and are quite similar. Generally, latency of the OFF response measure (E, F, and $G)$ did not appear to be shorter or longer than comparable $\mathrm{ON}$ response measures $(\mathrm{A}, \mathrm{B}$, and $\mathrm{C}$ ). All labeled $\mathrm{ON}$ and $\mathrm{OFF}$ amplitude and latency measures were plotted as a function of light flash intensity.

Linear regression analysis of all plots was then done by the method of least squares, using a General Linear Regression Program for the PDP 8/I computer (DECUS 8-118). Latency was found to decrease as luminance increased for most $\mathrm{ON}$ and OFF response measures. All ON latency measures yielded significant correlations at the .01 level except component D for Subjects K.F., and C.M.D., which yielded nonsignificant correlations. Correlation coefficients for the OFF latency measures reached significance for at least one component for each subject. OFF latency measure $G$ yielded a significant correlation (-.84) for Subject K.F. Significant correlations of -.93 and -.94 were found for OFF measures E and F, respectively, for Subject C.M.D. For Subject P.A.K., OFF measures $F$ and $H$ yielded significant correlation coefficients of -.93 and -.85 , 
respectively. Log-log functions were also fit to the latency-luminance data for all ON and OFF response measures. For each subject the log-log fits produced nearly identical significant correlation coefficients for the same ON and OFF latency measures as found significant for linear-log fits.

Within each subject, slopes of the linear regression lines for $\mathrm{ON}$ and OFF latency measures exhibiting similar latency ranges were compared. Only the regression lines yielding significant correlation coefficients for both $\mathrm{ON}$ and OFF latency measures were compared. For example, for Subject C.M.D., ON latency measure B and $\mathrm{OFF}$ latency measure $\mathrm{F}$ have similar latency ranges. Also, both measures yield significant correlation coefficients for Subject C.M.D. Meeting both these criteria, the linear regression slopes of measures $B$ and $F$ (for Subject C.M.D.) were compared (Figure 3). Figure 3 shows that within each subject the compared linear-log slopes are very similar. For each comparison a statistical test was run for significant differences between the regression coefficients (McNemar, 1969). For all intrasubject comparisons presented in Figure 3, no statistically significant differences were found between slopes of comparable ON and OFF latency measures.

Correlation coefficients for linear functions fitted to all labeled ON and OFF amplitude measures were also computed. Only amplitude measure B-C for the ON response yielded significant correlation coefficients for all subjects. Intrasubject comparisons of measures B-C and F-G were done for Subject C.M.D. only, since no significant correlations were found for any of the OFF amplitude measures for Subjects P.A.K. and K.F. In agreement with latency comparisons, there was no significant difference between amplitude slopes B-C and F-G for Subject C.M.D. Visual inspection of scattergrams did not reveal the obvious applicability of any nonlinear functions to the OFF amplitude data. Similar to findings on latency, log-log functions fitted the amplitude data as well as linear-log functions.

\section{DISCUSSION}

The results indicate that the deflections labeled $\mathrm{B}, \mathrm{C}$, and D for the ON response are similar to those in the OFF response labeled F, G, and $\mathrm{H}$. Although all subjects revealed an A deflection in the ON waveform, only Subject C.M.D. showed a comparable deflection in the OFF waveform. It is also noteworthy that deflection $\mathrm{H}$ of the OFF waveform appeared more variable than deflection D of the ON waveform, for all subjects, and overlapped poorly in latency range with measure $D$. The apparent similarities between ON and OFF VEBR waveform shapes reported in this study were not observed by Clynes et al. (1964). This difference in results may be related to differences in sample size. Clynes et al. (1964) compared ON and OFF VEBRs averraged from 300 EEG traces, while the VEBRs in this study were the sum of 1,000 EEG traces. Although a definitive study of sample size effects has not been done, it is clear that the need for larger sample sizes becomes more critical as the signal (VEBR) to noise (EEG plus VEBR) ratio decreases (Tepas, 1974). Since the OFF response is generally smaller in amplitude than the $\mathrm{ON}$, it is reasonable to suggest that a large sample is needed to allow equally reliable measurement and direct comparison of $\mathrm{ON}$ and $\mathrm{OFF}$ responses.

For all subjects, amplitude measure B-C of the ON response
SUBJECT: CMD

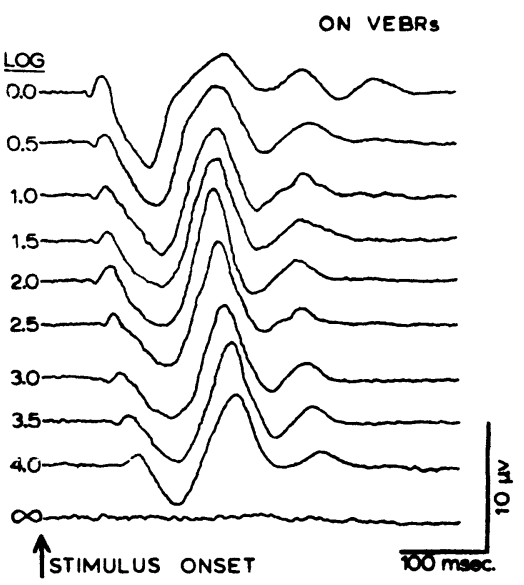

OFF VEBRS

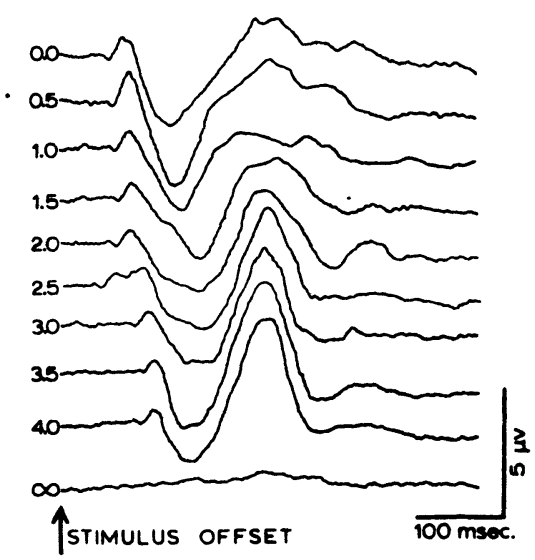

Figure 2. Averaged $O N$ and OFF VEBRs for one subject. Each waveform is a product of 1,000 flash onsets and offsets. The waveform labeled $\infty$ indicates EEG traces averaged when the constant blue surround was present, but no white flash (i.e., control trials). Note the different microvolt calibrations for each figure.

yielded a significant correlation coefficient when fitted by both linear-log and log-log regression lines. Tepas et al. (1974) have presented similar results for the ON amplitude measure B-C. They have noted that inadequate stimulus control can systematically diminish luminance effects on amplitude. Also, it is possible that the use of very high or very low luminances may result in nonlinear amplitude-luminance functions. Clynes et al. (1964) do not specify the deflection amplitude measures made, do not present their stimuli in Maxwellian view, nor do they report stimulus luminance levels administered. Thus, it is difficult to compare our results directly to those of Clynes et al. (1964).

In agreement with Tepas et al. (1974), latency of most ON response measures decreased as luminance increased, for all subjects. Latency-luminance plots for ON measures were adequately described by fitting either linear-log or log-log regression functions to the data. In contrast to changes in the ON component latencies, Clynes et al. (1964) reported that latency of the OFF response increased as luminance increased. The present study found opposite results for OFF latency measures for all three subjects. Clynes et al. (1964) did not report which latency deflections they measured, or whether a statistical analysis was done to determine the degree of relationship betwen latency and luminance.

Figure 3 illustrates the lack of statistically significant differences between the slopes of comparable significant ON and OFF luminance functions. The results demonstrate that the OFF 


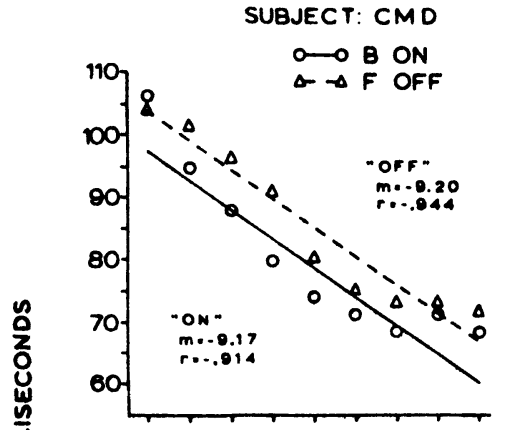

SUBJECT: KF

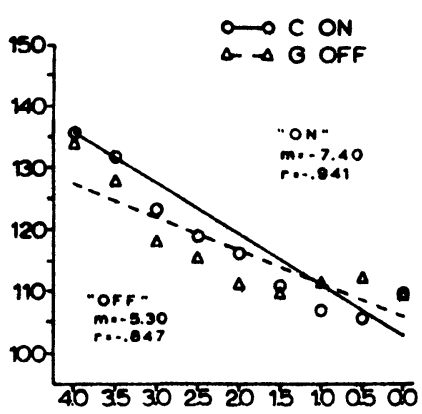

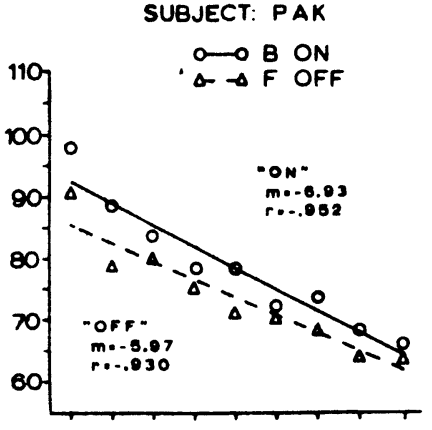

SUBJECT: PAK

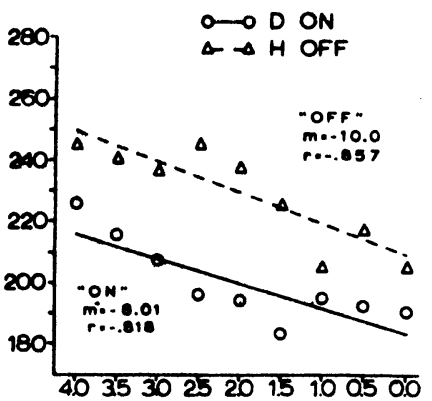

Figure 3. Latency-luminance functions for comparable measures of the $\mathrm{ON}$ and OFF VEBRs within each subject. Slope of the regression lines is abbreviated as " $\mathrm{m}$." The linear-log regression lines were fit to the mean data points using the method of least squares.

LOG UNITS OF NEUTRAL DENSITY FILTERING

VEBR sensitivity to luminance when significant linear-log or $\log -\log$ functions are fitted and compared. However, absolute differences in amplitude between simultaneously recorded $\mathrm{ON}$ and OFF response waveforms suggest a note of caution when equating them for sensitivity. Since amplitude is more related to luminance magnitude than latency, this implies that the $O N$ response would have to be considered more sensitive than the OFF. Nevertheless, the reliability of latency measures across luminance has been noted elsewhere (Wooten, 1972). Equivalent waveform shapes, latency ranges, and sensitivity to luminance within each subject, along with intrasubject response consistencies, emphasize the similarities between $O N$ and OFF reponses. Contrary to Clynes et al. (1964), our results illustrate symmetry between $\mathrm{ON}$ and $\mathrm{OFF}$ responses and suggest a common physiological system may mediate both responses. The same specific physiological system need not be involved in mediating both entirely. Indeed, differences in amplitude between the responses suggest that two similar but complimentary mechanisms could account for the results.

The findings of the present study suggest that the $\mathrm{ON}$ rather than the OFF VEBR be used as the dependent variable in studies assessing the effect of various stimulus parameters on VEBRs. Although particular measures of the OFF VEBR can be shown to vary with changes in luminance, the number of OFF measures showing this relationship are few compared to the ON VEBR. The fact that the OFF amplitude is smaller than the ON amplitude under similar conditions suggests that the ON VEBR is also more suitable for use in clinical settings. However, when attempting to record a VEBR from photophobic patients, the OFF response might be more easily tested than the $O N$ response.

\section{REFERENCES}

Bartlett, N. R., \& White, C. T. Cortical potentials evoked by the onset and termination of foveal stimulation. Psychonomic Science, 1968, 11, 357.

Clynes, M., Kohn, M., \& Lifshitz, K. Dynamics and spatial behavior of light evoked potentials, their modification under hypnosis, and on-line correlation of rhythmic components. Annals of the New York Academy of Science, 1964, 112, 468-508.

Efron, R. Artificial synthesis of evoked responses to light.
Annals of the New York Academy of Science, 1964, 112, 292-304.

EMRICh, H., \& MichaEL, D. Fourier analysis of on- and offeffects of evoked potentials. Electroencephalography and Clinical Neurophysiology, 1970, 29, 206-219.

FIsCHER, M. H. Electrobiologische Ersheinungen an der Hirnrinde. I. Pflugers Archive für die gesamte Physiologie des Menschen und der Tiere, 1932, 230, 161-178.

HARTER, M. R. Visually evoked cortical responses to the onset and offset of patterned light in humans. Vision Research, 1971, 2, 685-695.

JASPER, H. H. The ten-twenty electrode system of the international federation. Electroencephalography and Clinical Neurophysiology, 1958, 10, 371-375.

McNemar, Q. Psychological statistics. New York: Wiley, 1969.

PeAcock, S. M. Averaged "after-activity" and the alpha regeneration cycle. Electroencephalography and Clinical Neurophysiology, 1970, 28, 287-295.

SChweitzer, P. K., \& Tepas, D. I. Intensity effects on the auditory evoked brain response to stimulus onset and cessation. Perception \& Psychophysics, 1974, 4, 396-400.

TEPAS, D. I. Computer analysis of the electroencephalogram: Evoking, promoting and provoking. Behavior Research Methods \& Instrumentation, 1974, 6, 95-110.

Tepas, D. I., Gutteras, V., \& Klingaman, R. L. Variability of the human averaged evoked brain response to visual stimulation: A warning. Electroencephalography and Clinical Neurophysiology, 1974, 36, 533-537.

Tepas, D. I., Kress, G., \& Klingaman, R. L. APE: Average potential evaluation software for the LAB-8 system. Behavior Research Methods \& Instrumentation, 1975, 7, 120-124.

WestheImer, G. The Maxwellian view. Vision Research, 1966, 6, 669-682.

White, C. T., \& EAson, R. G. Evoked cortical potentials in relation to certain aspects of visual perception. Psychology Monographs, 1966, 80, 1-14.

Wooten, B. R. Photopic and scotopic contributions to the human visually evoked cortical potential. Vision Research, $1972,12,1647-1660$.

(Received for publication April 23, 1976.) 